Service social

\title{
Administration sociale et services sociaux, par Jules Perron, Chicoutimi, Gaëtan Morin, 1986, 285 pages.
}

\section{Georgette Béliveau et André Lajeunesse}

Volume 36, numéro 1, 1987

L'informatique dans les services sociaux

URI : https://id.erudit.org/iderudit/706349ar

DOI : https://doi.org/10.7202/706349ar

Aller au sommaire du numéro

Éditeur(s)

École de service social de l'Université Laval

ISSN

1708-1734 (numérique)

Découvrir la revue

Citer ce compte rendu

Béliveau, G. \& Lajeunesse, A. (1987). Compte rendu de [Administration sociale et services sociaux, par Jules Perron, Chicoutimi, Gaëtan Morin, 1986, 285 pages.]

Service social, 36(1), 166-169. https://doi.org/10.7202/706349ar d'utilisation que vous pouvez consulter en ligne.

https://apropos.erudit.org/fr/usagers/politique-dutilisation/ 
de façon plus spécifique, les expériences et les recherches qui ont cours dans ce domaine.

École de service social,

Martin Poulin

Université Laval.

Administration sociale et services sociaux, par Jules Perron, Chicoutimi, Gaëtan Morin, 1986, 285 pages.

Ce volume s'adresse à tous ceux qui désirent posséder un manuel de référence sur la gestion des services sociaux. Il se divise en trois parties: "Les fondements de l'administration sociale", "La dynamique de l'administration sociale", et "Quelques dimensions québécoises de l'administration sociale ".

L'auteur utilise une approche pédagogique et respecte les principes de base de la théorie de l'apprentissage : les chapitres sont bien présentés, faciles d'accès et se terminent par une synthèse faisant le pont avec le chapitre suivant ; les notions théoriques font ressortir les connaissances reconnues en Amérique du Nord et en Angleterre; le volume contient bon nombre de références et de citations. Il est illustré de tableaux et figures rendant les exposés plus concrets.

La première partie porte sur les fondements de l'administration sociale, et est composée de deux chapitres; l'un porte sur la notion d'administration sociale, l'autre sur la nature des services sociaux.

L'administration sociale est définie comme "un processus par lequel une société crée les services sociaux, les maintient, les développe et les incorpore à son organisation sociale" (p. 9). À partir de cette définition, l'auteur établit les liens qui unissent les politiques sociales et la pratique du service social à la gestion des services sociaux comme discipline reliée à un système de valeurs et soumise au respect de la personne humaine. Il émet le postulat que: "l'administration sociale n'est valide qu'à la condition d'humaniser les services sociaux et de maintenir l'équilibre entre la promotion de la personne humaine et la promotion de la société dans une idéologie de personnalisme social " (p. 37).

Le deuxième chapitre de cette première partie a pour principal objectif de fournir une clarification des termes employés en administration sociale, qu'il s'agisse de bien-être social, de services sociaux, de service social ou de besoin.

On y définit le bien-être social en termes de satisfaction des besoins essentiels des individus, d'atteinte de l'ordre social, de l'ensemble des mesures publiques et particulières d'ordre politique, juridique, économique, culturel et socio-sanitaire mises en place par la société en vue d'un développement intégral.

L'auteur retient, sur la notion de services sociaux, la définition de la Commission d'enquête sur la santé et le bien-être social du Québec, à savoir : " un ensemble de moyens de nature préventive, par lesquels la société vient en aide à des personnes, à des familles, à des groupes et à des collectivités, afin de les 
maintenir, de les intégrer ou de les réintégrer dans ce qu'ils considèrent comme une vie normale et de favoriser leur développement " (p. 47). Les quatre fonctions principales des services sociaux deviennent alors : adaptation personnelle, apprentissage et réapprentissage sociaux, organisation communautaire et régulation.

En le distinguant bien des "services sociaux" en général, Perron formule une définition du "service social", soit un "ensemble de connaissances et de technologies d'intervention sociale, qui a pour fonction de modifier réciproquement les interactions entre, d'une part, les personnes ou les collectivités et, d'autre part, leurs environnements, en vue de créer un équilibre dynamique des rapports sociaux qui soit favorable à la fois aux personnes concernées et à la société en général» (p. 54).

Cette définition est suivie d'un très bref rappel des principales méthodes du service social. Enfin, il présente les rapports entre le bien-être social (concept global), les services sociaux (programmes spécifiques) et le service social (discipline, modèle ou technologie).

On s'attarde ensuite au modèle systémique de distribution des services sociaux défini comme "un sous-système par lequel la société s'assure, par le canal d'établissements publics et privés, que les services sociaux prévus dans les politiques sociales se rendent avec une efficacité optimale aux individus et aux collectivités auxquels ils sont destinés" (p. 61).

Il faut également retenir que la distribution des services sociaux est étroitement liée à la structure organisationnelle ainsi qu'à la notion de besoin. Perron insiste sur la nécessité de maintenir une souplesse dans la définition de "besoin " qui impliquera une souplesse dans la programmation et la distribution des services.

En conclusion, cette première partie du volume, quoiqu'un peu abstraite et difficile, permet d'établir une base conceptuelle et donne une vue d'ensemble des notions utilisées en administration sociale.

La deuxième partie, sur la dynamique de l'administration sociale, est composée de trois chapitres. Le premier traite de l'apport des sciences administratives, le second fait référence à l'influence des sciences sociales, et le troisième est consacré à la gestion spécifique des services sociaux.

Cette section propose une intégration de la discipline et de l'art de l'administration sociale, pour les transformer en un instrument positif de gestion associant les variables reliées à la planification, à l'organisation, à la direction et au contrôle.

L'ampleur et l'accroissement des services sociaux entraînent l'obligation de bien administrer. Comme le type d'administration influence la quantité et la qualité des services offerts, il devient primordial de recourir à un modèle solide, adapté aux services sociaux. C'est ce que l'auteur tente d'élaborer en proposant l'adoption du schéma de Friedlander et Apte, qui divise en neuf fonctions les tâches administratives majeures d'une agence sociale publique ou privée: « rechercher des données, analyser des conditions sociales et des services requis pour faire face aux besoins, décider la meilleure manière pour atteindre les objectifs, planifier et distribuer les ressources, établir la structure organisationnelle 
et assigner les tâches, engager le personnel requis, surveiller et contrôler le personnel et les opérations financières, tenir les dossiers et rendre des comptes, obtenir les ressources financières." (p. 84).

L'auteur débute son analyse d'un modèle de gestion applicable à l'administration sociale en effectuant un rappel et une présentation sommaire de quelques processus administratifs. Ainsi, Fayol a mis en lumière les éléments de base de la gestion scientifique : planification, organisation, direction, contrôle. Newman imprègne ce modèle de base d'un mouvement dynamique et inclut la dimension des relations humaines. Laflamme se sert de la théorie des systèmes et met l'accent sur les valeurs, les motivations et les communications, tandis que Langevin, Tremblay et Bélanger introduisent la gestion participative par objectifs. Enfin, Kreitner développe et ajoute un modèle de contingence tenant compte de l'approche écologique et du "métamanagement ".

Perron présente ensuite certaines hypothèses contenues dans plusieurs modèles concernant la gestion des services sociaux.

En résumé, le développement continu des théories et des techniques de gestion des entreprises a influencé l'administration des services sociaux. II s'agit d'une inter-influence car la sociologie, la psychologie et les sciences du comportement ont pénétré l'administration. Il faut également noter la place importante occupée par l'approche systémique dans l'administration sociale.

La deuxième partie se termine sur la proposition d'un modèle dynamique de gestion des services sociaux qui s'inspire de l'approche systémique et y ajoute les techniques administratives de pointe pour proposer un modèle aux objectifs humanitaires maintenant l'appartenance communautaire et la responsabilité sociale.

L'auteur passe graduellement de la gestion des services sociaux au concept de gestion sociale. Il établit une différence entre la distribution des services sociaux, biens publics, à but non lucratif, avec des objectifs humanitaires et sensibles aux influences de l'environnement, et celle des biens de consommation.

Dans le premier chapitre de la troisième partie, qui expose quelques dimensions québécoises de l'administration sociale, Jules Perron présente, à l'aide d'une méthode d'analyse dite "systémique-institutionnelle", l'évolution des services sociaux québécois. Le second chapitre est consacré au système actuel de distribution des services sociaux. Enfin, dans le troisième, il effectue une analyse prospective et nous donne sa vision des enjeux à venir.

Il débute le chapitre sur l'histoire en nous présentant les documents à la base de ses recherches. Il accorde une place d'importance aux rapports et aux grandes commissions d'enquête, telles la commission Montpetit, la commission Tremblay, le rapport Boucher, la commission Castonguay-Nepveu ; il cite aussi des travaux internes à des établissements et des revues professionnelles. Sans apporter ici d'innovation, il utilise un matériel fiable et pertinent aux fins de son étude.

Dans la deuxième partie de ce chapitre, l'auteur explique sa méthode d'analyse dite "d'approche systémique-institutionnelle ». La dimension centrale 
à étudier, l'institution des services sociaux comme objet principal d'analyse, est appelée variable dépendante et est mise en relation avec les trois variables indépendantes suivantes : le politique, le socio-culturel et la religion. L'étude se fait ensuite par périodes : avant 1900, de 1900 à 1919, de 1920 à 1939, de 1940 à 1959, de 1960 à 1979.

Ce chapitre se termine par un excellent tableau-synthèse et par des commentaires judicieux découlant de sa lecture.

Le chapitre sur le système québécois de distribution des services sociaux commence par l'explication de sa philosophie et des principales mesures législatives présentement en vigueur. On y parle ensuite de l'organisation centrale, c'està-dire le Ministère de la santé et des services sociaux, et on nous explique, sous forme de différents tableaux, la mission sociale du gouvernement et les programmes de ce Ministère. Malheureusement, à cause des changements survenus dans la structure gouvernementale, dans les objectifs et les priorités, certains éléments présentés sont déjà dépassés. Suit une présentation du conseil régional de santé et des services sociaux, des établissements du réseau et du rôle des organismes bénévoles. Comme à l'habitude, l'auteur termine son travail par une excellente synthèse.

Le dernier chapitre débute par un résumé des principales caractéristiques des services sociaux québécois. L'auteur met ensuite l'accent sur les fondements philosophiques et sur les enjeux relatifs à la législation sociale et à la programmation, et termine en présentant, sous forme de planification prospective, sa vision personnelle de l'orientation future.

L'administration sociale est un art et une science qui diffère de l'administration proprement dite. Elle dépasse la technologie pure, possède des bases conceptuelles propres, une problématique spécifique et des objectifs. Le volet social est une force indispensable des systèmes de distribution des services sociaux. L'administration sociale est une discipline des sciences sociales vouée à l'évolution et destinée à faire progresser la société.

À travers cette philosophie personnelle, l'auteur a brossé le tableau des services sociaux québécois dans ses dimensions passée, présente et future et ce, avec succès.

Le volume, excellent et intéressant dans son ensemble, peut paraître difficile d'accès dans les premiers chapitres, mais possède le mérite de présenter, pour la première fois en français, les concepts de base de l'administration sociale adaptée aux services sociaux québécois, ce qui peut, par ailleurs, contribuer à limiter la portée de l'ouvrage. D'autre part, l'usage d'une excellente méthode pédagogique en fait un outil fort intéressant pour l'enseignement de la discipline du service social en milieu francophone.

École de service social,

Georgette BÉLIVEAU

Université Laval.

André LAJEUNESSE

Les établissements de santé

de la Vallée de la Matapédia. 